\title{
Organic Personality Syndrome
}

National Cancer Institute

\section{Source}

National Cancer Institute. Organic Personality Syndrome. NCI Thesaurus. Code C34872.

A mental disorder caused by intrinsic disease which is characterized by persistent alteration of a known behavioral pattern. Changes in emotional stability, motivation, judgment or impulse control are usually noted. It is broadly classified as a personality change due to a general medical condition. 